\title{
Globe
}

Revue internationale d'études québécoises

\section{Michel Venne [éd.], Penser la nation québécoise, Montréal, Québec Amérique, « Débats ", 2000, 309 p.}

\section{Daniel Salée}

Volume 3, numéro 2, 2000

URI : https://id.erudit.org/iderudit/1000596ar

DOI : https://doi.org/10.7202/1000596ar

Aller au sommaire du numéro

Éditeur(s)

Globe, Revue internationale d'études québécoises

ISSN

1481-5869 (imprimé)

1923-8231 (numérique)

Découvrir la revue

Citer ce compte rendu

Salée, D. (2000). Compte rendu de [Michel Venne [éd.], Penser la nation

québécoise, Montréal, Québec Amérique, « Débats ", 2000, 309 p.] Globe, 3(2),

208-213. https://doi.org/10.7202/1000596ar d'utilisation que vous pouvez consulter en ligne.

https://apropos.erudit.org/fr/usagers/politique-dutilisation/ 
hommage à l'inventeur d'un néologisme à fortune : "collectivisme ", mais comme le fait Angenot, qui, par le biais de cette figure exemplaire, pose " la question des marges du discours social [...] et du rôle des systèmes totaux » (p. 18), en démêlant un des écheveaux les plus embrouillés du XIXe siècle.

\section{Nadia Minerva \\ Université de Perugia (Italie)}

\section{Michel Venne [éd.], \\ Penser la nation québécoise \\ Montréal, Québec Amérique, «Débats », 2000, 309 p.}

Avec cet ouvrage, Michel Venne nous livre la version définitive d'une initiative qu'il avait lancée au cours de l'été 1999 dans les pages du journal Le Devoir où il est rédacteur en chef adjoint. On se souviendra qu'il avait alors invité une douzaine d'universitaires et d'intellectuels québécois à faire part de leur vision personnelle de la nation québécoise. Malgré les attraits de la saison estivale, la série d'articles connut un succès remarquable. En effet, chacun d'eux fut l'objet de commentaires nombreux et vigoureux, tant dans les pages du journal que sur son site internet. L'intérêt pour cette entreprise ne devait pas se démentir alors que quelques mois plus tard, en octobre, plus de 500 personnes prirent part à un colloque, organisé sous le patronage du Programme d'études sur le Québec de l'université McGill, au cours duquel les auteurs des textes parus dans Le Devoir débattirent ensemble et avec le public de leurs positions respectives. Penser la nation québécoise rassemble ces textes, revus et corrigés à la lumière des interventions du colloque, ainsi que quelques autres qui ne faisaient pas partie de la série originale. On y trouve au total seize essais - dix-sept, avec le texte d'introduction de Venne -, qui explorent à leur manière le défi que pose la coexistence sur le territoire québécois de la majorité francophone, d'une part, encore marquée dans le cadre juridico-politique canadien par son expérience historique de minoritaire dont elle cherche à s'affranchir, et de minorités linguistiques et ethnoculturelles, d'autre part, dont les 


\section{RECENSIONS}

impératifs théoriques de la démocratie moderne commandent qu'elles jouissent sans équivoque de tous les atouts de la citoyenneté. Comment " vivre ensemble dans la reconnaissance mutuelle de cette majorité et des minorités qui partagent le même territoire, sans nier nos origines?" (p. 13), c'est la question qui sert de prémisse à ce livre et que Michel Venne a posée à ses collaborateurs.

De façon générale, Penser la nation québécoise constitue un outil qui pourrait s'avérer utile à quiconque désire faire le point sur l'état des idées qui dominent actuellement les débats - universitaires, du moins - autour de la question nationale québécoise. L'aréopage impressionnant qu'a réuni Michel Venne regroupe presque tous les spécialistes reconnus de ce sujet (Charles Taylor, Gérard Bouchard, Daniel Jacques, Serge Cantin, Jocelyn Létourneau, Gilles Gagné, Gregory Baum, Marc Chevrier, Gilles Bourque, Jane Jenson, Danielle Juteau, Denys Delâge, Claude Bariteau, Michel Seymour, Jacques Beauchemin et Guy Rocher). Ceci dit, le produit final souffre des inévitables défauts qui affligent souvent les ouvrages du genre. Bien que la thématique retenue parvienne à insuffler une certaine cohérence à l'ensemble, la qualité des textes est inégale. Alors que certains se présentent sous une facture clairement académique, d'autres restent plus vagues ou carrément impressionnistes. Quelquesuns sont trop courts, à peine plus élaborés que dans la version parue dans Le Devoir. Sous cet aspect, l'ouvrage pourrait en décevoir plus d'un : certains auteurs qui nous avaient habitués à une pensée plus robuste nous servent ici les ersatz d'idées mieux exprimées ailleurs.

Penser la nation québécoise comporte toutefois d'autres travers infiniment plus problématiques. Michel Venne se targue d'avoir constitué « un ouvrage qui n'est pas l'expression d'un consensus entre les auteurs "; il confie même avoir été ouvert à ceux ou celles qui pourraient vouloir nier l'existence de la nation québécoise. Guy Rocher confirme sa démarche dans la postface en insistant sur la variété des sources et des référents disciplinaires. En réalité, la pluralité intellectuelle tant vantée n'est ni aussi profonde, ni aussi large que Venne et Rocher le prétendent. Par-delà les horizons disciplinaires distincts, par-delà les évidentes différences entre nationalistes québécois et fédéralistes canadiens, l'ouvrage est d'une 
homogénéité étonnante. La grande majorité des auteurs, adhérents de l'unité canadienne comme porte-drapeaux de la souveraineté politique du Québec, s'escriment en circonvolutions théoriques et programmatiques, marquées plus ou moins profondément au coin de la vertu démocratique; presque tous le font pour signifier, à divers degrés, l'incontournable nécessité d'incorporer la diversité ethnoculturelle à un projet ouvert et universel de construction de la nation québécoise; certains rappellent au surplus que le nationalisme québécois s'est depuis longtemps dédouané de ces relents ethnicistes tant honnis par ses ennemis politiques.

Le texte de Serge Cantin et, dans une moindre mesure, celui de Jacques Beauchemin enregistrent peut-être les seules dissonances dans ce concert foncièrement monocorde. Le premier s'inquiète de ce que " le nouveau crédo de la nation québécoise ouverte et plurielle, multiou transculturelle, mine le projet qu'il prétend servir, en le privant peu à peu de sa raison d'être... Car il implique que nous disparaissions par altruisme » (p. 92). Le second fustige la mauvaise conscience de ces intellectuels qui "sont devenus incapables de dire ce qui se donne pourtant comme une parfaite évidence, c'est-à-dire que le projet de la reconnaissance politique du Québec est porté par un groupe, et seulement lui, parfaitement repérable de ceux dont le parcours historique les a conduit à vouloir que leur nation s'appelle pays » (p. 260). Le texte de Cantin, sans doute le plus courageux et le plus vrai du livre, mais aussi le moins défendable moralement, tant il s'attache à une vision surannée de la société québécoise, fait pratiquement cavalier seul. À telle enseigne qu'on se demande s'il n'y est pas surtout pour illustrer ce à quoi il ne conviendrait plus d'associer la nation et le nationalisme québécois. À cette seule exception près, Michel Venne a manifestement choisi ses collaborateurs de manière à donner l'impression de transcender les divisions qui opposent tenants et adversaires du nationalisme québécois et, surtout, dans le but de faire progresser le débat sur la nation vers d'autres plans, plus inclusifs et novateurs. Au fond, il n'en est rien, car l'économie générale de l'ouvrage repose, d'un texte à l'autre, sur une façon quasi unitaire de saisir la nation québécoise et de la projeter sur un horizon sociopolitique qui ne dépasse guère en fin de compte le cadre plutôt étroit de la nation civique. De plus, cet 
horizon serait essentiellement contenu dans des paramètres - ceux, convenus, de la citoyenneté et de l'État libéraux - définis et portés au premier chef par la majorité francophone : on renie d'abord les positions d'un Serge Cantin - rectitude politique oblige - pour y revenir en bout de piste. Sont ainsi tenues à l'écart les voix qui n'auraient pas de la citoyenneté, du Québec et de la manière de s'y insérer, le même entendement. La chose étonne : il ne manque pourtant pas d'intellectuels immigrants, féministes ou minoritaires qui, eux aussi, pensent la nation québécoise. Ils le font, il est vrai, sur le mode de la transgression, du refus des rapports de pouvoir et de force imposés par l'État libéral, voire du rejet des critères patriarcaux et partiels de citoyenneté qui le définissent : un mode plutôt opposé à ce que Venne et ses collaborateurs préconisent.

Penser la nation québécoise propose donc beaucoup plus un portrait de famille aux discours convergents, à quelques nuances près, qu'une réflexion tous azimuts ouverte sur les possibles de la communauté politique québécoise. En fait, ce livre participe de la même urgence panique qui, depuis une dizaine d'années, s'est emparée des élites politiques et intellectuelles occidentales devant la " menace " de fragmentation identitaire et les remises en question de l'hégémonie socio-culturelle des classes et groupes sociaux dominants. Que faut-il faire pour apaiser la grogne des minoritaires sans perdre le contrôle de la situation, ni avoir l'air anti-démocratique? Voilà, décodée, la question que Michel Venne a réellement posée à ses collaborateurs. $\mathrm{La}$ véritable audace eût été de répondre que la nation moderne n'offre plus l'ancrage adéquat pour recomposer la communauté politique selon les impératifs nouveaux et mouvants de la démocratie. À l'évidence, ce n'est pas le genre de réponse que Michel Venne attendait - quoique Gregory Baum et Gilles Gagné esquissent quelques velléités en ce sens - et il aura choisi ses collaborateurs en conséquence. On ne trouvera donc dans son livre ni manière nouvelle de penser la nation québécoise, ni conception franchement novatrice de la communauté politique. On trouvera en lieu et place des poncifs de plus en plus éculés, ressassés depuis une décennie par ceux qui monopolisent le discours sur la question nationale québécoise avec, en prime, moult injonctions normatives au dialogue, à la concorde, au respect de la pluralité, etc., comme si la construction de la nation 
ne devait dépendre que de bonnes intentions et d'un œcuménisme politique bon teint.

Le questionnement qui anime la démarche de Michel Venne n'est pas illégitime. Seulement, il y en a d'autres, plus fondamentaux peutêtre, du moins plus intéressants du point de vue de l'analyse sociologique ou politique. Plutôt que de s'interroger sur les modalités idéales du vivre ensemble dans un contexte social pluraliste, ne vaudrait-il pas mieux se demander, par exemple, pourquoi cette nation que nationalistes territoriaux et civiques désirent tant tarde à advenir? De même, pourquoi faudrait-il privilégier la nation comme forme principale de communauté politique? Michel Venne n'a jamais fait secret de ses choix quant à l'avenir de la société québécoise et il serait injuste de lui tenir rigueur des interrogations qui le motivent. Mais, bien qu'il s'en défende, Penser la nation québécoise constitue d'abord et avant tout un ouvrage militant destiné à valoriser l'image d'ouverture intellectuelle et sociale que les nationalistes québécois de la dernière vague veulent faire accréditer, au Québec et ailleurs. Et, comme toutes les entreprises militantes, celle que nous propose Michel Venne n'a de valeur que si l'on accepte ses postulats. Or, ceux auxquels Venne et ses collaborateurs semblent souscrire ne sont pas sans poser problème. Leur discours sur la nation s'inscrit pour l'essentiel dans un cadre normatif et moral où elle est posée comme un idéal absolu de la communauté politique, comme forme inéluctable de vivre ensemble. Cette façon de voir les choses masque combien, à vrai dire, la nation constitue une fiction. Non pas au sens de la catégorie sociologique qu'elle représente sans doute, mais au sens du projet d'une communauté politique que l'on veut consensuelle, unie, universelle et intégratrice. Cela tient en grande partie à la quête obsessionnelle de cohésion sociale si caractéristique des sociétés libérales. Or la cohésion sociale, ça n'existe pas. Il peut bien y avoir apparence de cohésion dans une conjoncture donnée, mais cela n'est possible qu'au prix d'actes de pouvoir, de coercition, de répression idéologique et culturelle. Ainsi en est-il aussi de la nation comme forme en apparence stable et cohésive de communauté politique. Elle émerge en fait du mouvement contradictoire constant de domination et de résistance propre à toute société. Partant, il faut admettre que la nation civique, consensuelle et rationnelle, forgée à 


\section{RECENSIONS}

même une culture publique commune autour de laquelle on se rallierait d'emblée, ne peut exister, qu'elle est un pur produit de l'imagination philosophique. Ou alors, en supposant qu'elle soit possible, elle ne peut se construire qu'au prix de l'oblitération des revendications socio-économiques et des aspirations identitaires minoritaires.

C'est là une image dure et inconfortable de la nation? Peut-être. À tout prendre, elle semble préférable à la mythologie " bonne ententiste " si éloignée de la réalité sociale que proposent philosophes libéraux et nationalistes civiques, et dont l'ouvrage de Michel Venne est imprégné. Elle incite à aborder la nation froidement et à déconstruire l'acte de pouvoir qui l'a constituée à l'origine. La nation n'émane pas a priori du désir de vivre ensemble, mais doit être envisagée comme forme historiquement datée par laquelle s'est instituée la dynamique sociétale moderne. Voilà le fin mot de la démarche intellectuelle qui devrait nous animer. À terme, il est plus utile de bien comprendre la logique socio-politique dont participe la nation et par laquelle elle advient comme communauté politique, que de simplement penser la nation. Libre à ceux et celles qui préfèrent encore penser la nation de continuer à le faire, bien sûr. Qu'il soit clair cependant que leur effort contribuera sans doute peu ou prou à faire avancer notre compréhension des grandes modalités actuelles de transformation sociale et politique. Il n'aura de sens que dans les limites du projet politique nationaliste qui l'anime.

Daniel Salée Université Concordia 